\title{
NOTE ON THE GRADIENT OF THE GREEN'S FUNCTION*
}

\author{
BY G. C. EVANS
}

1. Integral of the Gradient of the Green's Function. The author, in $1929, \dagger$ mentioned without proof the fact that for a bounded open simply connected plane region $T$ the integral

$$
I(M)=\int_{T}|\nabla g(M, P)| d \sigma_{P},
$$

where $\nabla g(M, P)$ denotes the gradient of the Green's function with pole at $M$, is bounded, independently of the position of $M$ in $T$. A corresponding result holds for convex regions in three dimensions. It is the purpose of this note to bring out some of the differences between the two- and three-dimensional situations by a comparison of the analyses for the general plane simply connected region and the star-shaped three-dimensional region.

TheOREM 1. Let $T$ be a bounded open simply-connected region in the plane, $g(M, P)$ its Green's function with pole at $M$, and $I(M)=\int_{T}|\nabla g(M, P)| d \sigma_{P}$. Then

$$
I(M)<(2 \pi T g)^{1 / 2}\left(1-e^{-g}\right)^{-1},
$$

where $g$ is an arbitrary positive number and $T$ siands for meas. $T$; in particular

$$
I(M)<2.216(\pi T)^{1 / 2} .
$$

For a given $M$ the level curves of $g(M, P)$ are simple closed analytic curves, and neither $g(M, P)$ nor its gradient vanishes at a point of $T$. Denote by $h(M, P)$ the function conjugate to the Green's function, by $d s$ the element of arc of the level curve $s$ of $g(M, P)$, and by $d n$ the element of arc of the normal trajectory $n$, taken as positive in the direction towards $M$. The integral $I(M)$ is evidently convergent.

The Jacobian $J=d(x, y) / d(g, h)$ has the value

* Presented to the Society, August 31, 1932.

$\dagger$ Evans, Discontinuous boundary value problems of the first kind for Poisson's equation, American Journal of Mathematics, vol. 51 (1929), pp. 1-18. 


$$
\frac{d(x, y)}{d(g, h)}=\left(\frac{d n}{d g}\right)^{2}=\left(\frac{d s}{d h}\right)^{2}=|\nabla g(M, P)|^{-2},
$$

and if $u+v i=w(z)=e^{-(g+h i)}$ transforms the region $T$ into the interior of the unit circle in the $w$-plane, the value of the Jacob ian $J=d(x, y) / d(u, v)$ is

$$
\frac{d(x, y)}{d(u, v)}=\left|\frac{d z}{d w}\right|^{2}=\left(\frac{d n}{d g}\right)^{2}|w|^{-2}
$$

for $d w / d z=-w d(g+h i) / d z=-w(\partial g / \partial x-i \partial g / \partial y)$, and $d g / d n$ $=|d w / d z| /|w|$.

Hence if $\gamma$ is any positive number, $\rho_{1}=e^{-\gamma}$, and $\rho, \theta$ are polar coordinates in the w-plane,

$$
\begin{aligned}
I & =\int_{(\rho<1)} \frac{1}{|w|}\left|\frac{d z}{d w}\right| d u d w=\int_{0}^{1} d \rho \int_{0}^{2 \pi}\left|\frac{d z}{d w}\right| d \theta \\
& =I_{1}+I_{2},
\end{aligned}
$$

where

$$
\begin{aligned}
& I_{1}=\int_{(g<\gamma)}\left|\frac{d g}{d n}\right| d \sigma=\int_{\rho_{1}}^{1} f(\rho) d \rho, \\
& I_{2}=\int_{(g<\gamma)}\left|\frac{d g}{d n}\right| d \sigma=\int_{0}^{\rho_{1}} f(\rho) d \rho,
\end{aligned}
$$

with

$$
f(\rho)=\int_{0}^{2 \pi}\left|\frac{d z}{d w}\right| d \theta .
$$

Now by Schwarz's integral inequality, using (2), and writing $T$ for meas. $T$, we have

$$
I_{1}^{2} \leqq \int_{(g<\gamma)}\left(\frac{d g}{d n}\right)^{2} d \sigma \cdot \int_{(g<\gamma)} d \sigma<T \int_{(g<\gamma)} d g d h<2 \pi T \gamma
$$

and

$$
I_{1}<(2 \pi T \gamma)^{1 / 2}
$$

But by Hardy's well known theorem $f(\rho)$ is a non-decreasing function of $\rho$, for $d z / d w$ is holomorphic in $|w|<1$. Hence 


$$
I_{1} \geqq f\left(\rho_{1}\right)\left(1-\rho_{1}\right), I_{2} \leqq f\left(\rho_{1}\right) \rho_{1} .
$$

Consequently

$$
f\left(\rho_{1}\right) \leqq \frac{I_{1}}{1-\rho_{1}}<\frac{(2 \pi T \gamma)^{1 / 2}}{1-\rho_{1}}
$$

and

$$
I_{2}<(2 \pi T \gamma)^{1 / 2} \frac{\rho_{1}}{1-\rho_{1}}=(2 \pi T \gamma)^{1 / 2} \frac{e^{-\gamma}}{1-e^{-\gamma}}
$$

From (5) and $\left(5^{\prime}\right)$ we have (1), denoting $\gamma$ by $g$.

In particular, we may choose $g$ so as to make the right-hand member of (1) a minimum, for this expression is positive and becomes infinite as $g$ tends to 0 or $\infty$. In fact, the derivative of $g^{1 / 2}\left(1-e^{-g}\right)^{-1}$ vanishes at one and only one positive value of $g$, corresponding to the positive solution of the equation $e^{g}=1+2 g$. We calculate

$$
g=1.256, \rho_{1}=3.512^{-1}=.285,
$$

whence we have $\left(1^{\prime}\right)$. This completes the proof of the theorem.

In particular, if $T$ is a circle and $M$ is at the center, we have $I=2(\pi T)^{1 / 2}$, by direct calculation, a result which is near enough to $\left(1^{\prime}\right)$ to suggest an interesting problem in the calculus of variations. For the circle of unit radius, $M$ being distant $a$, $a<1$, from the center, we have $I=\pi\left[\left(1-a^{2}\right) / a\right] \log [(1+a)$ $/(1-a)]$, and in fact $I$ is maximum when $a=0$.

2. Corollaries. The following remarks follow as corollaries. The integral $I(M)$, from (2), takes the forms

$$
I(M)=\int_{0}^{\infty} s_{g} d g=\int_{0}^{2 \pi} n_{h} d h,
$$

so that again, each of these quantities is bounded, independently of the position of $M$, in accordance with the theorem.

Moreover $s_{g}$ is an increasing function of $\rho=e^{-g}$.* In fact, $s_{o}=\int|d z / d w| d(\rho \theta)=\rho f(\rho)$, which is strictly increasing, since $f(\rho)$ is non-decreasing. From (5) therefore, $g s_{g}<I_{1}<(2 \pi T g)^{1 / 2}$ and

* W. Seidel, Über die Ränderzuordnung bei konformen Abbildungen, Mathematische Annalen, vol. 104 (1931), pp. 182-243. 


$$
s_{g}<\left(\frac{2 \pi T}{g}\right)^{1 / 2}=\left(\frac{2 \pi T}{-\log \rho}\right)^{-1 / 2} .
$$

Similarly, from the inequality on $f(\rho)$, we have the sharper inequality

$$
s_{g}<(2 \pi T g)^{1 / 2}\left(e^{g}-1\right)^{-1} .
$$

Finally, $f(\rho)=2 \pi s_{g} / c_{\rho}$, where $c_{\rho}$ is the circumference of the circle of radius $\rho=e^{-g}$, and therefore

$$
I=2 \pi \int_{0}^{1} \frac{s_{g}}{c_{\rho}} d \rho,
$$

where $s_{g} / c_{\rho}$ is a non-decreasing function of $\rho$.

3. Three-Dimensional Case. For a three-dimensional region $T$, which is homeomorphic with the interior of a sphere, the function $g(M, P)$ is defined by the methods of Harnack, but its gradient may vanish at some interior point even if the boundary of $T$ is normal with respect to the Dirichlet problem. $\dagger$ The level surfaces would not then necessarily be homeomorphic with the surface of a sphere. For the theorem which follows, we restrict ourselves merely, however, to a region $T$, bounded and homeomorphic with the interior of a sphere.

TheOREm 2. Let $F$ be the set of points $M$ of $T$ from which $T$ is star-shaped. Then $F$ is null or closed with respect to $T$. Let $I(M)=\int_{T}|\nabla g(M, P)| d \tau_{P}$. Then for all points of $F$

$$
I \leqq 4.76\left(\pi^{2} T\right)^{1 / 3} \leqq 4 \pi g^{-1}+(4 \pi g T)^{1 / 2},
$$

where $g$ is an arbitrary positive number.

In particular, if $T$ is convex, $F \equiv T$ and (9) holds for all points of $T$.

We prove that $F$ is closed with respect to $T$ by proving that the complementary set in $T$, namely $T-F$, is open. Let $M$ be a point of $T-F$. Then, by definition, there is a point $P$ of $T$ such that the segment $M P$ contains a point, say $Q$, not in $T$. Consider the lines through $Q$, and let $\tau_{M}, \tau_{P}$ be spherical regions,

* J. J. Gergen, Mapping of a three dimensional region on a sphere, American Journal of Mathematics, vol. 52 (1930), pp. 197-224.

$\dagger \mathrm{J} . \mathrm{J}$. Gergen, Note on the Green function of a star-shaped three dimensional region, American Journal of Mathematics, vol. 53 (1931) pp. 746-752. 
with centers $M$ and $P$ and radii $\rho_{M}$ and $\rho_{P}$, respectively, small enough to lie entirely in $T$. Given $\rho_{P}$ we can choose $\rho_{M}$ small enough so that every line through $Q$ and a point of $\tau_{M}$ passes through a point of $\tau_{P}$. This will be the case if $\rho_{M}<\rho_{P} \cdot(M Q / Q P)$. Hence every point of $T-F$ is an interior point of $T-F$.

4. Proof of Second Part of Theorem 2. In order to prove the remainder of the theorem, let $T^{\prime}$ be a bounded simply-connected region whose boundary is normal with respect to the Dirichlet problem, and let $M$ be a point of $T^{\prime}$ such that $\nabla g_{T^{\prime}}(M, P)$ does not vanish for $P$ in $T^{\prime}$; this will be the case, in particular, if $T^{\prime}$ is star-shaped from $M$. Such a region may be mapped on the interior of a unit sphere in a one-one manner in such a way that the level surface $g(M, P)=g$ goes into the concentric sphere of radius $\rho=1 /(1+g)$, and the normal trajectories to the level surfaces go into the radii of the sphere; the mapping is not conformal, but on the other hand, again unlike the plane case, it reduces to the identical transformation as $\rho$ tends to zero.*

Hence if we consider the cone of trajectories abutting on an element $d S_{g}$ of the $g$-surface, which subtends a solid angle $d \omega$ at $M$ and the corresponding element of surface $d C_{\rho}$ on the sphere of radius $\rho=1 /(1+g)$, we have

$$
\frac{d g}{d n} d S_{g}=d \omega=-\frac{d g}{d \rho} d C_{\rho} ; \quad \frac{d S_{o}}{d C_{\rho}}=-\frac{d n}{d \rho},
$$

instead of $d S_{\theta} / d C_{\rho}=(d n / d \rho)^{2}$, which would be the case if the transformation were conformal. We have

$$
\begin{aligned}
& J=\frac{d\left(n, S_{g}\right)}{d(g, \omega)}=\left(\frac{d n}{d g}\right)^{2}, \\
& J_{1}=\left|\frac{d\left(n, S_{g}\right)}{d(\rho, \omega)}\right|=\frac{1}{\rho^{2}}\left(\frac{d n}{d g}\right)^{2}=\frac{1}{\rho^{2}} \frac{d n}{d g} \frac{d S_{g}}{d \omega},
\end{aligned}
$$

and

$$
I(M)=\int_{T^{\prime}} \frac{d g}{d n} d \tau=\int_{(0<\theta<\infty)} \frac{d n}{d g} d g d \omega=4 \pi \int_{0}^{1} \frac{S_{g}}{C_{\rho}} d \rho,
$$

* J. J. Gergen, American Journal of Mathematics, vol. 52, loc. cit. The identity $(d g / d n) d S_{g}=d \omega$ is established by Gergen in the as yet unpublished part of his Thesis (Rice Institute, 1928). With the mapping already set up the demonstration is not difficult. 
with similar forms for the two integrals $I_{1}=\int_{(g<\gamma)}(d g / d n) d \tau$ and $I_{2}=\int_{(\sigma>\gamma)}(d g / d n) d \tau, \gamma$ being a positive number.

Analogously to the plane case, we have

$$
I_{1}^{2}<T^{\prime} \int_{(\theta<\gamma)}\left(\frac{d g}{d n}\right)^{2} d \sigma=T^{\prime} \int_{(\theta<\gamma)} d g d \omega=4 \pi \gamma T^{\prime}
$$

consequently

$$
I_{1}<(4 \pi \gamma T)^{1 / 2} .
$$

But for the treatment of $I_{2}$ we cannot follow the earlier method, since $f(\rho)=4 \pi S_{g} / C_{\rho}$ is not now necessarily a non-decreasing function of $\rho$.

In fact, if $T^{\prime}$ is a sphere of radius $a$ and $r=\left(x^{2}+y^{2}+z^{2}\right)^{1 / 2}$, $M$ being at the origin, we have $g(M, P)=1 / r-1 / a$, and corresponding values of $r$ and $\rho$ are given by the relation $1 / r-1 / a$ $=1 / \rho-1$, so that

$$
\frac{S_{g}}{C_{\rho}}=\frac{r^{2}}{\rho^{2}}=\left(1+\frac{1-a}{a} \rho\right)^{-2},
$$

which is an increasing function of $\rho$ if $a>1$, but a decreasing function if $a<1$. In the plane, the corresponding expression for two circles is independent of $\rho$.

Let us suppose, however, that $T^{\prime}$ is star-shaped from $M$. In this case the $g$-surfaces are homeomorphic with spherical surfaces, are analytic and enclose regions which are star-shaped from $M$; moreover*

$$
|\nabla g(M, P)|>g / r, \quad r=M P .
$$

But $g(M, P)=1 / r-v$, where $v$ is a harmonic function positive in $T^{\prime}$, so that $1 / r=v+g>g$; consequently

It follows, then, that

$$
|\nabla g(M, P)|>g^{2} \text {. }
$$

$$
\begin{aligned}
I_{2} & =\int_{(g>\gamma)} \frac{d g}{d n} d T=\int_{(g>\gamma)}\left(\frac{d g}{d n}\right)^{-1} d \omega d g \\
& <4 \pi \int_{\gamma}^{\infty} g^{-2} d g=4 \pi \gamma^{-1} .
\end{aligned}
$$

\footnotetext{
* J. J. Gergen, American Journal of Mathematics, vol. 53, loc. cit.
} 
This, with the inequality already obtained for $I_{1}$, gives

$$
I<4 \pi \gamma^{-1}+\left(4 \pi \gamma T^{\prime}\right)^{1 / 2}, \quad \gamma>0,
$$

for all points $M$ from which $T^{\prime}$ is star-shaped. The minimum value of this expression occurs for $\gamma=2(2 \pi / T)^{1 / 3}$, from which the value in the second member of (9) is obtained, namely, $I<3\left(4 \pi^{2} T^{\prime}\right)^{1 / 3}$.

We note, incidentally, that from the values of $d S_{v}$ and $d n$, already given, we obtain

$$
I(M)=\int_{0}^{\infty} S_{g} d g=\int_{(4 \pi)} N_{\omega} d \omega,
$$

$S_{0}$ being the total area of the $g$-surface and $N_{\omega}$ the total length of the trajectory originating at $M$ in the angular element $d \omega$.

5. Boundary of $T$ not Normal. We may pass now to the theorem for $T$, whose boundary may not be normal for the Dirichlet problem. It may be remarked, nevertheless, that the presence of an irregular boundary point very much restricts the nature of the set $F$.

Let $M$ be a point of $F, r=M P, Q$ the point where the ray $r$ cuts the unit spherical surface with center $M, P^{\prime}$ the first point where it cuts the closed boundary set $\bar{T}-T$. Then (following Gergen) $r^{\prime}=M P^{\prime}$ is a lower semi-continuous function of $Q$ on the unit sphere, $r^{\prime}=f(Q)$. We have, then, $f(Q)=\lim _{n=\infty} f_{n}(Q)$, where the $f_{n}(Q)$ may be chosen as a strictly increasing sequence of continuous functions; in fact, the choice may be made so that we have $f_{n+1}(Q)-f_{n}(Q)>1 / n-1 /(n+1)=1 /\left(n^{2}+n\right)$. For $n$ great enough the regions $T_{n}: r<f_{n}(Q)$ contain $M$ and are (evidently) star-shaped from $M$. But each $f_{n}(Q)$ may be replaced by a function $\phi_{n}(Q)$, as regular as we please, such that uniformly $\left|f_{n}(Q)-\phi_{n}(Q)\right| \leqq 1 /\left(2 n^{2}+2 n\right)$; in fact, such a function $\phi_{n}(Q)$ may be obtained by averaging $f_{n}(Q)$, more than once if necessary, over a circular neighborhood of $Q$ on the unit sphere, the radius of the neighborhood being a constant $\delta$ chosen suitably small.*

Thus we obtain a strictly increasing sequence $\phi_{n}(Q)$ which defines a sequence of regions $T_{n}^{\prime}$, such that $\bar{T}_{n}^{\prime}$ is contained in

* For the properties of approximations in average consult H. E. Bray, Proof of a formula for an area, this Bulletin, vol. 29 (1923), pp. 264-270. 
$T_{n-1}^{\prime}$. These regions are star-shaped from $M$, have boundaries which are regular with respect to the Dirichlet problem and, for $n$ great enough, contain any given closed subset of $T$. In fact, $\lim _{n=\infty} \phi_{n}(Q)=f(Q)$. If $g_{n}(M, P)$ denotes the Green's function for $T_{n}^{\prime}$, we have, uniformly in any closed subset $K$ of $T-M$,

$$
\begin{aligned}
& \lim _{n=\infty} g_{n}(M, P)=g(M, P), \\
& \lim _{n=\infty}\left|\nabla g_{n}(M, P)\right|=\nabla g(M, P), \quad P \text { in } K .
\end{aligned}
$$

But for $n$ large enough so that $K$ is contained in $T_{n}^{\prime}$, we have

$$
\int_{K}\left|\nabla g_{n}(M, P)\right| d \tau_{P}<4 \pi \gamma^{-1}+\left(4 \pi \gamma T_{n}^{\prime}\right)^{1 / 2}<4 \pi \gamma^{-1}+(4 \pi \gamma T)^{1 / 2},
$$

$\gamma$ an arbitrary positive number. Hence

$$
\int_{K}|\nabla g(M, P)| d \tau_{P} \leqq 4 \pi \gamma^{-1}+(4 \pi \gamma T)^{1 / 2}
$$

and finally,

$$
\int_{T}|\nabla g(M, P)| d \tau_{P} \leqq 4 \pi \gamma^{-1}+(4 \pi \gamma T)^{1 / 2} .
$$

This completes the proof of Theorem 2 .

For the sphere of radius $R$, with $M$ at the center, we calculate

$$
I=4 \pi R=2\left(6 \pi^{2} T\right)^{1 / 3}=3.63\left(\pi^{2} T\right)^{1 / 3} .
$$

The Rice Institute 